\title{
Analysis of model for the transmission dynamics of Zika with sterile insect technique
}

\author{
Usman A. Danbaba, Salisu M. Garba
}

\begin{abstract}
A deterministic model for the transmission dynamics of Zika, that takes into account the aquatic and non-aquatic stages of mosquito development is constructed and rigorously analysed. The model with fraction of male mosquitoes being sterilized assumed direct (human-human) and indirect (human-mosquito-human) transmission. Stability analysis of the equilibria and sensitivity analysis of parameters associated with the computed reproduction number were presented. Numerical simulation were carried out to support the analysis.
\end{abstract}

\footnotetext{
Usman A. Danbaba

Department of Mathematics and Applied Mathematics, University of Pretoria, Pretoria DST-NRF Centre of Excellence in Mathematical and Statistical Sciences (CoE-MaSS) South Africa

e-mail:u.a.danbaba@gmail.com

Salisu M. Garba

Department of Mathematics and Applied Mathematics, University of Pretoria, Pretoria South Africa

e-mail: salisu.garba@up.ac.za
}

Citation: Usman A. Danbaba, Salisu M. Garba, Analysis of model for the transmission dynamics of Zika with sterile insect technique, in R. Anguelov, M. Lachowicz (Editors), Mathematical Methods and Models in Biosciences, Biomath Forum, Sofia, 2018, pp. 81-99, http://dx.doi.org/10.11145/texts.2018.01.083

Copyright: (C) 2018 Danbaba et al. This article is distributed under the terms of the Creative Commons Attribution License (CC BY 4.0) which permits unrestricted use, distribution, and reproduction in any medium, provided the original author and source are credited. 


\section{Introduction}

Zika fever is a mosquito-borne Flavivirus caused by Zika virus ( $\mathrm{Zv}$ ). The virus was first identified in Uganda in 1947, through a monitoring network of sylvatic yellow fever in rhesus monkeys. Five years later, human infection was identified in Uganda and Tanzania. Since then, Zika outbreaks have been recorded in Africa, Americas, Asia and the Pacific [9, 16, 20]. Zv is primarily transmitted by Aedes mosquito, mainly Aedes aegypti (which also transmits Yellow fever, Dengue, Chikungunya, West Nile, and Japanese encephalitis viruses) [20, 23]. However, sexual transmissions between humans have also been reported [9, 19].

Controlling mosquito population is one of the most important tool in the control and/or prevention of vector borne diseases. Sterile insect technique is a promising and non polluting method of vector control, it has been thought of in the control of mosquito borne diseases [29]. In this work, we attempt to investigate the impact of sterilization on vector control and disease transmission, by considering the interaction of both mosquito (where fraction of males are sterilized) and human population, this allows us to assess the potential impact of sterilization.

\section{Model formulation}

Although complex, life cycle of mosquito is generally divided into two stages; aquatic and non-aquatic. The aquatic stage is denoted by a single compartment $A$, (this is similar to the assumption in other models in the literature, such as those in [1, 11, 13, 14, 17] and some of the references therein).

The non-aquatic stage is divided into seven compartments, consisting of young females $(Y)$, fertilized non-sterilized females (those who could lay eggs and hatch due to mating with non-sterile male mosquitoes) $\left(F_{N}\right)$, fertilized sterilized females (those who could lay eggs but do not hatch due to mating with sterile male mosquitoes) $\left(F_{S}\right)$, non-sterilized fertilized infected females $\left(F_{N I}\right)$, sterilized fertilized infected females $\left(F_{S I}\right)$, and non-sterilized male mosquitoes $\left(M_{N}\right)$. Sterilized male mosquitoes $\left(M_{S}\right)$ are introduced into the population at a constant rate. Thus, the total mosquito population is given by

$$
N_{V}(t)=A(t)+Y(t)+F_{N}(t)+F_{S}(t)+F_{N I}(t)+F_{S I}(t)+M_{N}(t)+M_{S}(t) .
$$

Human population is divided into susceptible $\left(S_{H}\right)$, infected $\left(I_{H}\right)$ and recovered humans $\left(R_{H}\right)$. The total human population is given by

$$
N_{H}(t)=S_{H}(t)+I_{H}(t)+R_{H}(t) .
$$




\subsection{Incidence function}

The incidence in human and mosquito populations are respectively, given by

$$
\lambda_{H}=\beta_{1} \frac{\left(F_{N I}+\eta_{1} F_{S I}\right)}{N_{V}}+\beta_{2} \frac{\left(I_{H}+\eta_{2} R_{H}\right)}{N_{H}}
$$

and

$$
\lambda_{V}=\beta_{3} \frac{I_{H}}{N_{H}},
$$

where, $\beta_{1}\left(N_{H}, N_{V}\right)=\rho_{M H} \xi_{1}$ is the adequate contact rate of infectious mosquito with susceptible human. Probability that contact is made with an infectious mosquito is given by $\frac{1}{N_{V}}\left(F_{N I}+\eta_{1} F_{S I}\right)$, with $\eta_{1} \in(0,1)$ as a modification parameter. $\beta_{2}=$ $\rho_{H H} \xi_{2}$ is the adequate contact (sexual) rate of infectious human with susceptible human, $\frac{1}{N_{H}}\left(I_{H}+\eta_{2} R_{H}\right)$ is the probability that contact is made with an infectious human (while $\eta_{2} \in(0,1)$ is a modification parameter). $\beta_{3}=\rho_{H M} \xi_{3}$ is the adequate contact rate of an infectious human with a susceptible female mosquito, $\frac{I_{H}}{N_{H}}$ is the probability that contact was made with an infectious human.

For number of bites to be conserved, the total number of bites made by mosquitoes must be equal to the total number of bites received by humans [2, 3, 21]. Thus, the following equation holds

$$
\beta_{1}\left(N_{H}, N_{V}\right) N_{H}=\beta_{3} N_{V}
$$

Therefore,

$$
\lambda_{H}=\frac{\beta_{3}\left(F_{N I}+\eta_{1} F_{S I}\right)+\beta_{2}\left(I_{H}+\eta_{2} R_{H}\right)}{N_{H}} .
$$

\subsection{Dynamics of mosquito population}

The aquatic stage $(A)$ involves egg, larva and pupa. The population is increased from oviposition by reproductive mosquitoes at the rate $\phi$. It reduces due to natural death at the rate $\mu_{V}$, it is assumed that natural death occurs in all mosquito compartments (except sterilized male mosquitoes) at the rate $\mu_{V}$, density dependence death at the rate $\mu$, they mature and move out of aquatic stage at the rate $b_{V}$. So that,

$$
\frac{d A}{d t}=\phi F_{N I}+\phi F_{N}-\mu A^{2}-\mu_{V} A-b_{V} A
$$

Mosquitoes at the non-aquatic stage are considered adult. Young female mosquito evolve from aquatic stage at the rate $r b_{V} A$, they mate with non-sterilized male mosquitoes and progress to the compartment of fertilized, non-sterilized susceptible female mosquitoes $\left(F_{N}\right)$ at the rate $\alpha_{N} \frac{M_{N}}{M}$, or with a sterilized male mosquito and move to the compartment of fertilized, sterilized susceptible female mosquito 
$\left(F_{S}\right)$, at the rate $\alpha_{S} \frac{M_{S}}{M}$. Therefore, this gives

$$
\frac{d Y}{d t}=r b_{V} A-\frac{\alpha_{N} M_{N}}{M} Y-\frac{\alpha_{S} M_{S}}{M} Y-\mu_{V} Y .
$$

Fertilized, non-sterilized susceptible female mosquitoes are generated from compartment $Y$ by mating with a non-sterilized male mosquito $\left(M_{N}\right)$. In order to nourish their eggs before oviposition, they need blood, they will probably bite an infectious human and move to the compartment of fertilized, non-sterilized infectious female mosquitoes $\left(F_{N I}\right)$ at the rate $\lambda_{V}$, or they bite a susceptible human and stay in the compartment, in which case, they can lay eggs. They die naturally at the rate $\mu_{V}$. So that,

$$
\frac{d F_{N}}{d t}=\frac{\alpha_{N} M_{N}}{M} Y-\lambda_{V} F_{N}-\mu_{V} F_{N}
$$

Similarly, the compartment of fertilized, sterilized susceptible female mosquitoes are generated through the fertilization of young female mosquitoes by sterilized male mosquitoes. This population is reduced by infection they acquired following contact with infectious humans and progress to the $F_{S I}$ compartment at a rate $\lambda_{V}$. This gives

$$
\frac{d F_{S}}{d t}=\frac{\alpha_{S} M_{S}}{M} Y-\lambda_{V} F_{S}-\mu_{V} F_{S}
$$

Fertilized, infectious non-sterilized female mosquitoes are generated by the infection of mosquitoes in $F_{N}$ class, and are decreased by natural death. Hence,

$$
\frac{d F_{N I}}{d t}=\lambda_{V} F_{N}-\mu_{V} F_{N I}
$$

A fertilized, infectious sterilized female mosquito population is generated from $F_{S}$ after biting an infectious human. Thus,

$$
\frac{d F_{S I}}{d t}=\lambda_{V} F_{S}-\mu_{V} F_{S I}
$$

Non-sterilized male mosquitoes evolve directly from aquatic stage at the rate $(1-$ $r) b_{V}$, and decreased due to natural death, so that,

$$
\frac{d M_{N}}{d t}=(1-r) b_{V} A-\mu_{V} M_{N}
$$

Sterilized male mosquitoes $\left(M_{S}\right)$ are released into the population at rate $\omega(t)$ at time $t$. However, due to some environmental and geographical factors that may affect the mixing of sterilized and wild mosquitoes, such as location of mosquito breeding site, it is convenient to assume that, only a fraction $p$ of the released mosquitoes will join wild mosquito population, likewise, because of differences in the physiology of wild and sterilized mosquitoes, a parameter $g$ is used to capture the mean mating competitiveness of sterilized mosquito, so that the actual number of sterilized male mosquitoes competing with wild mosquitoes is $\frac{M_{S}}{p g}$, and therefore, the growth rate 
of sterilized male mosquitoes is $p g \omega(t)$. This formulation is similar to that in [1]. The population is reduced due to natural death at the rate $\mu_{M}$. Hence,

$$
\frac{d M_{S}}{d t}=\operatorname{pg} \omega(t)-\mu_{M} M_{S}
$$

\subsection{Dynamics of human population}

The population of susceptible humans are generated by birth or immigration at a constant rate $b_{H}$. This population is decreased by acquiring infection after receiving substantive amount of bites capable of disease transmission from an infectious mosquito $\left(F_{N I}\right.$ or $\left.F_{S I}\right)$ at a rate $\lambda_{H 1}$. However, due to recent developments on the potentiality of human-to-human transmission [9, 18], we assumed a direct (horizontal) transmissions from an infected human to a susceptible human at a rate $\lambda_{H 2}$, and they are reduced by natural death at a rate $\mu_{H}$. It is worth mentioning that, Zika is the first Flavivirus known to be transmitted sexually from an infectious human to a susceptible human [19]. They are represented by

$$
\frac{d S_{H}}{d t}=b_{H}-\lambda_{H} S_{H}-\mu_{H} S_{H}
$$

The population of infectious human is generated by infection of susceptible humans at the rate $\lambda_{H}$, and decreases due to recovery (at a rate $\gamma$ ), natural death at the rate $\mu_{H}$ and disease induced death at a rate $\delta$, so that,

$$
\frac{d I_{H}}{d t}=\lambda_{H} S_{H}-\delta_{H} I_{H}-\gamma I_{H}-\mu_{H} I_{H}
$$

Finally, the population of recovered individuals is generated by the recovery of infected individuals (at a rate $\gamma$ ) and reduces due to natural death at a rate $\mu_{H}$. This gives

$$
\frac{d R_{H}}{d t}=\gamma I_{H}-\mu_{H} R_{H}
$$

It is worth mentioning that, high viral load was found in the semen and saliva of recovered patients weeks after recovery, hence, there is high chance of human-tohuman vaginal or oral sex transmission by a recovered human [9, 16, 18].

\subsection{Model equation}

Similar to the formulation in [1], we assumed that, the mating competitiveness of both sterilized and non-sterilized mosquitoes are equal, that is $\alpha_{S}=\alpha_{N}=\alpha$. Furthermore, since there are only two mating possibilities, either with a sterilized or non-sterilized mosquitoes, we let $\frac{M_{S}}{M_{N}+M_{S}}=\theta$, so that $\frac{M_{N}}{M_{N}+M_{S}}=1-\theta$. A flow chart 
for the model is illustrated in Figure 1 .

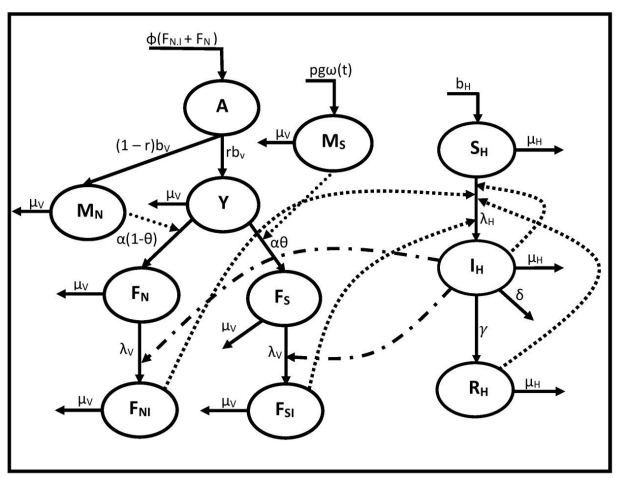

Fig. 1: Flow diagram of mosquito-human interaction

Therefore, Zika transmission model is given by the following system of non-linear differential equations with the description of the parameters and variables given in Tables 1 and 2):

$$
\text { 舜 }\left\{\begin{array}{l}
\frac{d S_{H}}{d t}=b_{H}-\lambda_{H} S_{H}-\mu_{H} S_{H}, \\
\frac{d I_{H}}{d t}=\lambda_{H} S_{H}-\delta I_{H}-\gamma I_{H}-\mu_{H} I_{H}, \\
\frac{d R_{H}}{d t}=\gamma I_{H}-\mu_{H} R_{H},
\end{array}\right.
$$

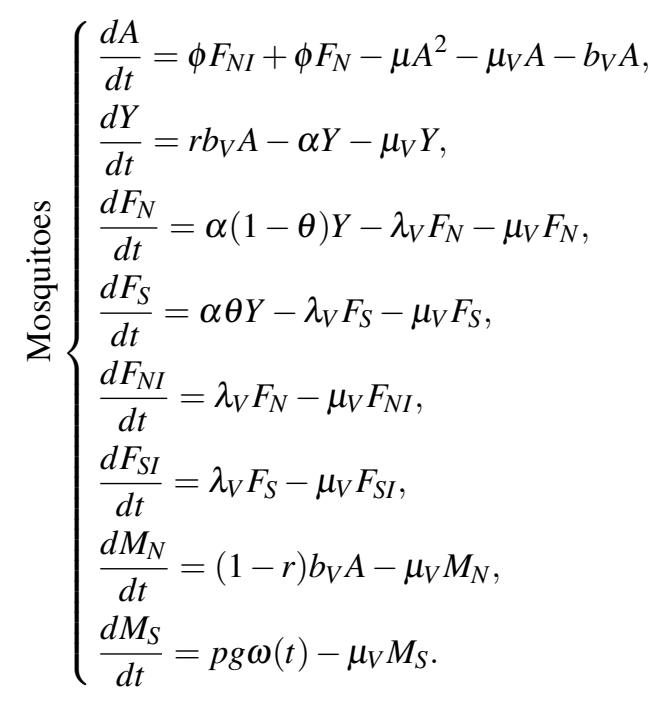


Table 1: Two sets of parameter values used in numerical simulations, with low baseline values that give $R_{0}=0.1489<1$, while $R_{0}=4.7033>1$ for the high baseline values

\begin{tabular}{|c|c|c|c|c|}
\hline Parameters & Range & Low baseline & High baseline & References \\
\hline$r$ & $(0,1)$ & 0.5 & 0.5 & $\mid 14]$ \\
\hline$\delta$ & 0.001 & 0.001 & 0.001 & {$\left[\begin{array}{ll|}8 & 21]\end{array}\right.$} \\
\hline$\theta$ & $(0,1)$ & 0.2 & 0.4 & assumed \\
\hline$\alpha$ & $(0,1)$ & 0.7 & 0.7 & [14] \\
\hline$\mu$ & 0.00001 & 0.00001 & 0.00001 & 11 \\
\hline$\phi$ & $100-200$ & 100 & 120 & [7] \\
\hline$b_{H}$ & 30 & 30 & 30 & |2] \\
\hline$b_{V}$ & $0.05-0.1$ & 0.05 & 0.08 & \begin{tabular}{|l|l|l|}
12 & 13 & 14
\end{tabular} \\
\hline$\eta_{1}, \eta_{2}$ & $(0,1)$ & 0.5 & 0.5 & assumed \\
\hline$\gamma$ & $0.059-0.167$ & 0.14 & 0.08 & |27] \\
\hline$\xi_{1}$ & $0.3-1$ & 0.3 & 0.5 & |24 25 \\
\hline$\xi_{2}$ & $0.01-0.20$ & 0.02 & 0.05 & $\mid 25]$ \\
\hline$\xi_{3}$ & $0.3-1$ & 0.3 & 1 & 24.25 \\
\hline$\mu_{V}$ & $0.043-0.25$ & 0.2 & 0.09 & $|\overline{20}| \overline{24} \mid$ \\
\hline$\mu_{H}$ & 0.00004 & 0.00004 & 0.00004 & | 12 - $13.14 \mid$ \\
\hline$\rho_{H H}$ & $0-1$ & 0.02 & 0.04 & 20.25 \\
\hline$\rho_{M H}$ & $0.1-0.75$ & 0.2 & 0.7 & 20 \\
\hline$\rho_{H M}$ & $0.5-1$ & 0.55 & 0.75 & 20,24 \\
\hline
\end{tabular}

The last equation of (5) is independent of other compartments and has the following solution

$$
M_{S}(t)=e^{-\mu_{M} t}\left(M_{S}(0)+\int_{0}^{t} e^{\mu_{M} j} p g \omega(j) d j\right) .
$$

\subsection{Basic properties}

Lemma 1. The solution of model (5) uniquely exists. In addition it is positive and bounded.

Proof. The right hand side of model $[5]$ is $C^{1}$ in $\mathbb{R}_{+}^{10}$, hence solution exists locally and it is unique.

It is clear from (5) that, if $S_{H}=0$, then $S_{H}^{\prime}=b_{H}>0$. Similarly, if $A=0$, then $A^{\prime}=\phi\left(F_{N I}+F_{N}\right)>0$ provided $F_{N I}>0$ and $F_{N}>0$. In general, for all $x_{i}=0$, $f_{i}=x_{i}^{\prime}>0$ whenever $x_{j}>0$ (monotone increasing with respect to $x_{j}$ ), so that, by Proposition B.7. of [30], solution to the system is positive. Also,

$$
\frac{d N_{H}}{d t}=b_{H}-\gamma I_{H}-\mu_{H} N_{H} \leq b_{H}-\mu_{H} N_{H} .
$$

The equation of aquatic mosquitoes population can be considered as having logistic growth, with carrying capacity $\mathscr{K}$. So that, $A(t) \leq \mathscr{K}$. 
Table 2: Sensitivity index of $R_{0}$ with respect to parameters of the model (5) for $R_{0}=0.5606<1$ and $R_{0}=13.0160>1$ using the values of Table 1

\begin{tabular}{|c|c|c|c|}
\hline Parameter & Description & Low base sensitivity & High base sensitivity \\
\hline$\vec{r}$ & $\begin{array}{l}\text { Modification parameter for matura- } \\
\text { tion rate of mosquitoes }\end{array}$ & +0.45294 & +0.38821 \\
\hline$\delta$ & Disease induced death rate of humans & -0.00551 & -0.00995 \\
\hline$\theta$ & $\begin{array}{l}\text { Average mating rate of sterilized male } \\
\text { mosquito }\end{array}$ & -0.06773 & -0.14846 \\
\hline$\alpha$ & Mating capability of male mosquitoes & +0.10065 & +0.04423 \\
\hline$\mu$ & $\begin{array}{l}\text { Density dependent death rate of } \\
\text { aquatic mosquitoes }\end{array}$ & -0.22277 & -0.19334 \\
\hline$\phi$ & $\begin{array}{l}\text { Oviposition rate of fertilized female } \\
\text { mosquitoes }\end{array}$ & +0.23017 & +0.19488 \\
\hline$b_{H}$ & Recruitment rate of humans & -0.22277 & -0.19334 \\
\hline$b_{V}$ & Maturation rate of mosquitoes & +0.45146 & +0.38766 \\
\hline$\eta_{1}$ & $\begin{array}{l}\text { Modification parameter for transmis- } \\
\text { sion of sterilized infectious mosquito }\end{array}$ & +0.03640 & +0.06621 \\
\hline$\eta_{2}$ & $\begin{array}{l}\text { Modification parameter for the trans- } \\
\text { mission of recovered human }\end{array}$ & +0.55053 & +0.61179 \\
\hline$\gamma$ & Recovery rate of humans & -0.22097 & -0.18453 \\
\hline$\beta_{2}$ & $\begin{array}{l}\text { Transmission rate of infectious hu- } \\
\text { mans to susceptible humans }\end{array}$ & +0.55446 & +0.61332 \\
\hline$\beta_{3}$ & $\begin{array}{l}\text { Transmission rate of infectious hu- } \\
\text { mans to susceptible mosquito }\end{array}$ & +0.44554 & +0.38668 \\
\hline$\mu_{V}$ & Natural death rate of mosquitoes & -0.78228 & -0.62677 \\
\hline$\mu_{H}$ & Natural death rate of humans & -0.32798 & -0.41886 \\
\hline
\end{tabular}

Let the total mosquito population at the non-aquatic stage be given by

$$
N_{M}=Y+F_{N}+F_{S}+F_{N I}+F_{S I}+M_{N}
$$

Then,

$$
\frac{d N_{M}}{d t} \leq b_{V} \mathscr{K}-\mu_{V} N_{M}
$$

Applying Gronwall lemma on (7) and (8) gives

$$
N_{H}(t) \leq N_{H}(0) e^{-\mu_{H}}+\frac{b_{H}}{\mu_{H}}\left(1-e^{-\mu_{H}}\right)
$$

and

$$
N_{M}(t) \leq N_{M}(0) e^{-\mu_{V}}+\frac{b_{V}}{\mu_{V}} \mathscr{K}\left(1-e^{-\mu_{V}}\right),
$$

which are bounded and therefore solutions exists for all $t \geq 0$.

Theorem 1. The following biologically-feasible region of the model (5) is positivelyinvariant 


$$
\begin{aligned}
& \left\{\left(S_{H}, I_{H}, R_{H}, A, Y, F_{N}, F_{S}, F_{N I}, F_{S I}, M_{N}\right) \in \mathbb{R}_{+}^{10}\right. \\
& \left.\quad: S_{H}+I_{H}+R_{H} \leq \frac{b_{H}}{\mu_{H}}, A \leq \mathscr{K}, Y+F_{N}+F_{S}+F_{N I}+F_{S I}+M_{N} \leq \frac{b_{V}}{\mu_{V}} \mathscr{K}\right\},
\end{aligned}
$$

where $\mathscr{K}$ is the carrying capacity of the aquatic compartment.

Proof. It is clear from (9) and (10) that, $N_{H}(t) \leq \frac{b_{H}}{\mu_{H}}$ whenever $N_{H}(0) \leq \frac{b_{H}}{\mu_{H}}$. Similarly, $N_{M}(t) \leq \frac{b_{V}}{\mu_{V}} \mathscr{K}$ if $N_{V}(0) \leq \frac{b_{V}}{\mu_{V}} \mathscr{K}$. Consequently, every solution initiated in $\Omega$ remain in $\Omega$ for all time $t \geq 0$.

\section{Existence and stability of equilibria}

Let

$$
N_{0}=\frac{\phi r b_{V} \alpha(1-\theta)}{\left(b_{V}+\mu_{V}\right)\left(\alpha+\mu_{V}\right) \mu_{V}}
$$

be defined as the basic offspring number of the mosquito population. It is the average number of offspring produced by a single female mosquito that mated with a nonsterilized male mosquito in its entire lifespan.

\subsection{Disease-free equilibrium}

Let $N_{0}>1$, that is, on average, a reproductive female mosquito reproduces more than one offspring in her entire life span, then there exist a non trivial, positive disease free equilibrium denoted by $E_{0}$, such that,

$$
\begin{aligned}
E_{0} & =\left(S_{H}^{*}, I_{H}^{*}, R_{H}^{*}, A^{*}, Y^{*}, F_{N}^{*}, F_{S}^{*}, F_{N I}^{*}, F_{S I}^{*}, M_{N}^{*}\right) \\
& =\left(\frac{b_{H}}{\mu_{H}}, 0,0, A^{*}, \frac{r b_{V} A^{*}}{K_{3}}, \frac{(1-\theta) \alpha r b_{V} A^{*}}{K_{3} \mu_{V}}, 0,0, \frac{\theta \alpha r b_{V} A^{*}}{K_{3} \mu_{V}}, \frac{(1-r) b_{V} A^{*}}{K_{3} \mu_{V}}\right),
\end{aligned}
$$

where

$$
\left(A^{*}\right)^{2}+\frac{K_{2}}{\mu}\left(1-N_{0}\right) A^{*}=0, \text { therefore } A^{*}=\frac{K_{2}}{\mu}\left(N_{0}-1\right) .
$$

\subsubsection{Local stability of $E_{0}$}

The local stability of the DFE $\left(E_{0}\right)$ can be established using the next generation operator. The $F$ matrix is given by 


$$
F=\left(\begin{array}{cccc}
\beta_{2} & \eta_{2} \beta_{2} & \beta_{3} & \eta_{1} \beta_{3} \\
0 & 0 & 0 & 0 \\
\beta_{3} \frac{F_{N}^{*}}{N_{H}^{*}} & 0 & 0 & 0 \\
\beta_{3} \frac{F_{S}^{*}}{N_{H}^{*}} & 0 & 0 & 0
\end{array}\right)
$$

while the $V$ matrix is

$$
V=\left(\begin{array}{cccc}
K_{1} & 0 & 0 & 0 \\
-\gamma & \mu_{H} & 0 & 0 \\
0 & 0 & \mu_{V} & 0 \\
0 & 0 & 0 & \mu_{V}
\end{array}\right)
$$

Therefore using the method described in [10], the next generation matrix (NGM) with large domain $K_{L}$ is given by

$$
K_{L}=\left(\begin{array}{cccc}
\frac{\beta_{2}\left(\mu_{H}+\eta_{2} \gamma\right)}{K_{1} \mu_{H}} & \frac{\beta_{2} \eta_{2}}{\mu_{H}} & \frac{\beta_{3}}{\mu_{V}} & \frac{\beta_{3} \eta_{1}}{\mu_{V}} \\
0 & 0 & 0 & 0 \\
\beta_{3} \frac{F_{N}^{*}}{N_{H}^{*}} & 0 & 0 & 0 \\
\beta_{3} \frac{F_{S}^{*}}{N_{H}^{*}} & 0 & 0 & 0
\end{array}\right),
$$

so that the next generation matrix is a $3 \times 3$ matrix given by

$$
K=\left(\begin{array}{ccc}
\frac{\beta_{2}\left(\mu_{H}+\eta_{2} \gamma\right)}{K_{1} \mu_{H}} & \frac{\beta_{3}}{\mu_{V}} & \frac{\beta_{3} \eta_{1}}{\mu_{V}} \\
\beta_{3} \frac{F_{N}^{*}}{N_{H}^{*}} & 0 & 0 \\
\beta_{3} \frac{F_{S}^{*}}{N_{H}^{*}} & 0 & 0
\end{array}\right) .
$$

Following [10, 15], the associated reproduction number of the system model (5) denoted by $\mathscr{R}_{0}$ is given by

$$
\mathscr{R}_{0}=\frac{1}{2}\left(\mathscr{R}_{H H}+\sqrt{\mathscr{R}_{H H}^{2}+4 \mathscr{R}_{H V} \mathscr{R}_{V H}}\right),
$$

where

$$
\mathscr{R}_{H H}=\frac{\beta_{2}\left(\mu_{H}+\eta_{2} \gamma\right)}{K_{1} \mu_{H}}, \quad \mathscr{R}_{H V}=\frac{\beta_{3} b_{V} \mu_{H} r}{K_{1} K_{3} b_{H}}
$$

and

$$
\mathscr{R}_{V H}=\frac{\beta_{3} K_{2} \alpha\left[\theta \eta_{1}+(1-\theta)\right]\left(N_{0}-1\right)}{\mu_{V}^{2} \mu}
$$

are the reproduction numbers associated with Zika transmission from human to human, human to vector and vector to human, respectively.

Lemma 2. The disease-free equilibrium $\left(E_{0}\right)$, of model (5) with (2) and (4) is locally asymptotically stable if $\mathscr{R}_{0}<1$, and unstable if $\mathscr{R}_{0}>1[15]$. 


\subsection{Interpretation of $\mathscr{R}_{0}$}

In the absence of direct transmission, the threshold quantity $\left(\mathscr{R}_{1}\right)$ defined by

$$
\begin{aligned}
\mathscr{R}_{1} & =\sqrt{4 \mathscr{R}_{V H} \mathscr{R}_{H V}} \\
& =\sqrt{\frac{\beta_{3}^{2} b_{V} \alpha r K_{2}\left(N_{0}-1\right)\left[\theta \eta_{1}+(1-\theta)\right]}{N_{H}^{*} K_{1} K_{3} \mu_{V}^{2} \mu}}
\end{aligned}
$$

is the expected number of secondary cases generated by an infected case introduced into a population, consisting of completely susceptible. It is interpreted as follows. Susceptible humans get infection from an infectious mosquito $\left(F_{N I}\right.$ or $\left.F_{S I}\right)$. The number of infections caused by non-sterilized infectious mosquitoes (near DFE) is given by the product of its infection rate $\left(\frac{\beta_{3}}{N_{H}^{*}}=\frac{\beta_{3} \mu_{H}}{b_{H}}\right)$, with the average duration in the infectious (non-sterilized) class $\left(\frac{1}{\mu_{V}}\right)$, and the probability that a female mosquito survives the fertilized non-sterilized class $\left(F_{N}\right)$, and move to fertilized non-sterilized and infectious compartment $\left(\frac{\alpha(1-\theta)}{\mu_{V}}\right)$ thus (noting that $S_{H}^{*}=\frac{b_{H}}{\mu_{H}}$ )

$$
\frac{\beta_{3} \mu_{H} \alpha(1-\theta)}{b_{H} \mu_{V}^{2}} S_{H}^{*}=\frac{\beta_{3} \alpha(1-\theta)}{\mu_{V}^{2}} .
$$

Similarly, the number of human infections generated by sterilized mosquitoes (near $\mathrm{DFE}$ ) is given by the product of the infection rate of sterilized infectious mosquitoes $\left(\frac{\beta_{3} \eta_{1}}{N_{H}^{*}}=\frac{\beta_{3} \eta_{1} \mu_{H}}{b_{H}}\right)$, with the average duration in the infectious (sterilized) class $\left(\frac{1}{\mu_{V}}\right)$, and the probability that a female mosquito survives the fertilized and sterilized class $\left(F_{S}\right)$, and move to fertilized, sterilized and infectious compartment $\left(\frac{\alpha \theta}{\mu_{V}}\right)$, so that

$$
\frac{\beta_{3} \mu_{H} \alpha \theta \eta_{1}}{b_{H} \mu_{V}^{2}} S_{H}^{*}=\frac{\beta_{3} \alpha \theta \eta_{1}}{\mu_{V}^{2}} .
$$

Therefore, the sum of (16) and (17) gives the average number of new human infections generated by infectious mosquitoes (sterilized or non-sterilized).

$$
\mathscr{R}_{V H}=\frac{\beta_{3} \alpha(1-\theta)}{\mu_{V}^{2}}+\frac{\beta_{3} \alpha \theta \eta_{1}}{\mu_{V}^{2}}=\frac{\beta_{3} \alpha\left[\theta \eta_{1}+(1-\theta)\right]}{\mu_{V}^{2}} .
$$

Mosquito infection is caused by infectious humans only, the number of mosquitoes infection caused by infectious human (near the DFE), is the product of its infection rate $\left(\frac{\beta_{3}}{N_{H}^{*}}=\frac{\beta_{3} \mu_{H}}{b_{H}}\right)$, with the average duration in infectious class $\frac{1}{K_{1}}$, hence (with $\left.Y^{*}=\frac{A^{*} b_{V} r}{K_{3}}\right)$,

$$
\mathscr{R}_{H V}=\frac{\beta_{3}}{N_{H}^{*} K_{1}} Y^{*}=\frac{\beta_{3} b_{V} \mu_{H} r}{K_{1} K_{3} b_{H}} .
$$


The geometric mean of (18) and (19) gives the associated reproduction number (since $A^{*}=\frac{K_{2}\left(N_{0}-1\right)}{\mu}$ )

$$
\mathscr{R}_{1}=\sqrt{\frac{\beta_{3}^{2} b_{V} \alpha r K_{2}\left(N_{0}-1\right)\left[\theta \eta_{1}+(1-\theta)\right]}{N_{H}^{*} K_{1} K_{3} \mu_{V}^{2} \mu}} .
$$

The number of new human-human infections (sexual), generated by an infectious human $\left(I_{H}\right)$ (near DFE) is given by the product of infection rate of infectious human $\left(\frac{\beta_{2} S_{H}^{*}}{N_{H}^{*}}\right)$, and the average duration in the infectious class $\left(\frac{1}{K_{1}}\right)$, this gives

$$
\frac{\beta_{2}}{K_{1}}
$$

Similarly, the number of new human infections (sexual) generated by a recovered human, is given by the product of its infectious rate $\left(\frac{\beta_{2} \eta_{2} S_{H}^{*}}{N_{H}^{*}}\right)$, the probability that human survives the infectious stage $\left(\frac{\gamma}{K_{1}}\right)$ and move to recovered class, and the average duration in the recovered class $\left(\frac{1}{\mu_{H}}\right)$, this gives

$$
\frac{\beta_{2} \eta_{2} \gamma}{K_{1} \mu_{H}}
$$

Hence, the sum of (20) and (21) gives the threshold quantity associated with the human-human Zika transmission

$$
\mathscr{R}_{H H}=\frac{\beta_{2}}{K_{1}}+\frac{\beta_{2} \eta_{2} \gamma}{K_{1} \mu_{H}}=\frac{\beta_{2}\left(\mu_{H}+\eta_{2} \gamma\right)}{K_{1} \mu_{H}} .
$$

Thus, the associated reproduction number for the Zika model with direct transmission is given by

$$
\mathscr{R}_{0}=\frac{1}{2}\left(\mathscr{R}_{H H}+\sqrt{\mathscr{R}_{H H}{ }^{2}+4 \mathscr{R}_{V H} \mathscr{R}_{H V}}\right) .
$$

It is worth mentioning that, $\mathscr{R}_{0}$ is consistent with those obtained by Brauer et al [2] and Chitnis et al [6], Gao et al [20] for epidemic models of vector borne diseases with direct or vertical transmissions.

\subsection{Endemic equilibrium}

Let

$$
E_{1}=\left(S_{H}^{* *}, I_{H}^{* *}, R_{H}^{* *}, A^{* *}, Y^{* *}, F_{N}^{* *}, F_{S}^{* *}, F_{N I}^{* *}, F_{S I}^{* *}, M_{N}^{* *}\right)
$$


represents an arbitrary positive endemic equilibrium point (EE) of the model (5). Furthermore, let

$$
\lambda_{H}^{* *}=\frac{\beta_{3}\left(F_{N I}^{* *}+\eta_{1} F_{S I}^{* *}\right)+\beta_{2}\left(I_{H}^{* *}+\eta_{2} R_{H}^{* *}\right)}{S_{H}^{* *}+I_{H}^{* *}+R_{H}^{* *}}
$$

and

$$
\lambda_{V}^{* *}=\beta_{3} \frac{I_{H}^{* *}}{S_{H}^{* *}+I_{H}^{* *}+R_{H}^{* *}}
$$

be the associated forces of infections. Solving equations of model (5) at the steady state gives

$$
\begin{aligned}
S_{H}^{* *} & =\frac{b_{H}}{\lambda_{H}^{* *}+\mu_{H}}, \quad I_{H}^{* *}=\frac{\lambda_{H}^{* *} b_{H}}{K_{1}\left(\lambda_{H}^{* *}+\mu_{H}\right)}, \\
R_{H}^{* *} & =\frac{\lambda_{H}^{* *} b_{H} \gamma}{K_{1} \mu_{H}\left(\lambda_{H}^{* *}+\mu_{H}\right)}, \quad A^{* *}=\frac{K_{2}}{\mu}\left(N_{0}-1\right), \\
Y^{* *} & =\frac{r b_{V}}{K_{3}} A^{* *}, \quad F_{N}^{* *}=\frac{b_{V} r \alpha(1-\theta)}{K_{3}\left(\lambda_{V}^{* *}+\mu_{V}\right)} A^{* *}, \\
F_{S}^{* *} & =\frac{b_{V} r \alpha \theta A^{* *}}{K_{3}\left(\lambda_{V}^{* *}+\mu_{V}\right)}, \quad F_{N I}^{* *}=\frac{b_{V} \lambda_{V}^{* *} r \alpha(1-\theta) A^{* *}}{K_{3} \mu_{V}\left(\lambda_{V}^{* *}+\mu_{V}\right)} \\
F_{S I}^{* *} & =\frac{b_{V} \lambda_{V}^{* *} r \alpha \theta A^{* *}}{K_{3} \mu_{V}\left(\lambda_{V}^{* *}+\mu_{V}\right)}, \quad M_{N}^{* *}=\frac{(1-r) b_{V} A^{* *}}{\mu_{V}} .
\end{aligned}
$$

\section{Sensitivity analysis}

Sensitivity analysis is a tool used in studying the variation of an output of a model due to change in the input parameters. Using elasticity index, we perform local sensitivity analysis (where all other parameters are held at a certain baseline) for the basic reproduction number $\left(\mathscr{R}_{0}\right)$. The method is used to measure the percentage change of a parameter say $\alpha$, with respect to a percentage change of a quantity say $\mathscr{R}_{0}(\alpha)$. The basic reproduction number $\left(\mathscr{R}_{0}\right)$ can be used to measure the potential impact of a disease. The normalized sensitivity index (elasticity indices) of $\mathscr{R}_{0}(\alpha)$ with respect to $\alpha$ is [5]

$$
\Upsilon_{\alpha}^{\mathscr{R}_{0}}=\frac{\partial \mathscr{R}_{0}}{\partial \alpha} \times \frac{\alpha}{\mathscr{R}_{0}} .
$$

Using data in Table 1, we give in Table 2 the sensitivity index of the parameters for low and high baseline values. For both low and high transmission regions, $\mathscr{R}_{0}$ is most negatively correlated to $\mu_{V}$, with $\Upsilon_{\mu_{V}}^{\mathscr{R}_{0}}=-0.78228$ in low region and $\Upsilon_{\mu_{V}}^{\mathscr{R}_{0}}=$ -0.62677 . Similarly, $\mathscr{R}_{0}$ is most positively correlated to $\beta_{2}$ in both cases. 


\section{Numerical simulations}

The Zika model (5) is simulated using parameter values in Table 1 together with the following initial conditions

$$
\begin{aligned}
& S_{H}(0)=600, I_{H}(0)=20, R_{H}(0)=0, \\
& A(0)=2400, Y(0)=500, F_{N}(0)=300, F_{S}(0)=100, \\
& F_{N I}(0)=100, F_{S I}(0)=50, M_{N}(0)=150
\end{aligned}
$$

Low baseline values give $\mathscr{R}_{0}=0.5606<1$, while high baseline values give $\mathscr{R}_{0}=$ $13.0160>1$. Fig. 2 illustrates the solution profile of the model (5), showing cumulative number of new human cases with different values of $\theta$ (probability of a female mosquito mating with a sterilized male). For $\alpha=0.7$ and $\theta=0.2$, the rate of sterilization $(\alpha \theta)=0.14$, that is $(20 \%)$ of young female mosquitoes mate with sterilized male mosquitoes, the percentage increase (which depends on $\theta$ ) is negatively correlated to the cumulative number of new human cases.

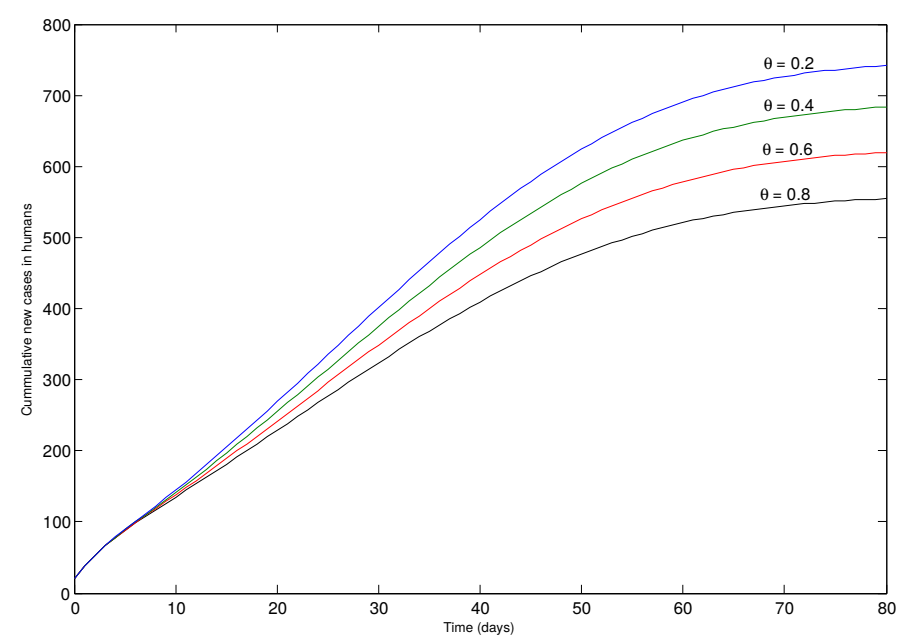

Fig. 2: Simulation of (5) showing cumulative number of new cases in humans with different values of $\theta$

Fig. 3 and Fig. 4 show population of infected humans with different initial conditions. Fig. 3 shows the convergence to a non zero equilibrium when $\mathscr{R}_{0}=$ $13.0160>1$, while Fig. 4 shows the convergence to DFE of infected humans when $\mathscr{R}_{0}=0.5606<1$. Disease prevalence (ratio of infected humans by the total human population), for both high and low baseline values are respectively shown in Fig. 5 and Fig. 6 Fig. 5 shows convergence of solution to endemic equilibrium, while Fig. 6 shows convergence to DFE with different initial conditions. 


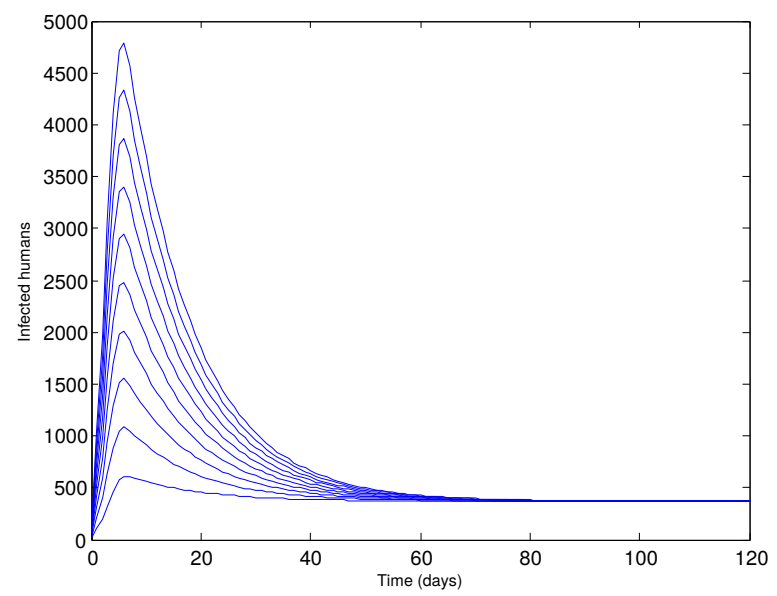

Fig. 3: Simulation of (5) showing infected humans with different initial conditions and $R_{0}=13.0160$.

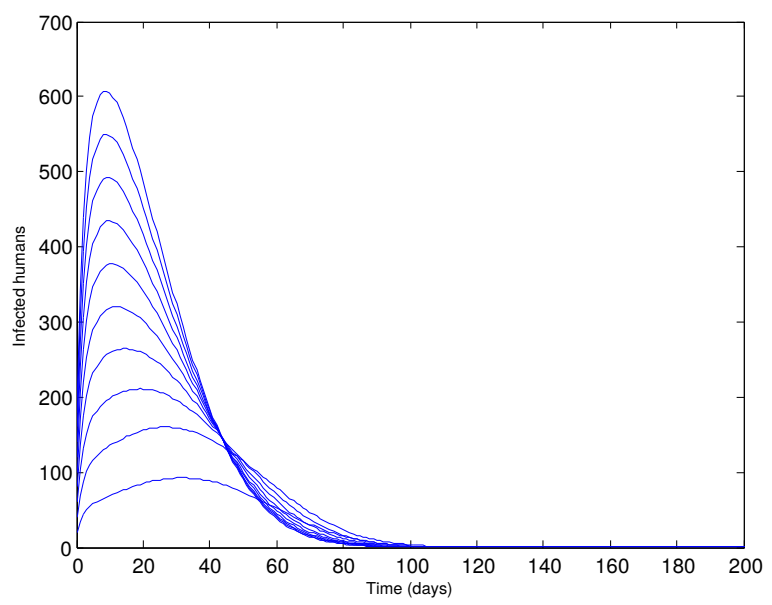

Fig. 4: Simulation of (5) showing infected humans with different initial conditions and $R_{0}=0.5606$.

Fig. 7 depicts the solution profile of the model (5) showing total number of reproductive mosquitoes, with different values of $\theta$, such that, for $\theta=0.2$, the population spikes four times and faded, while the number of spike reduces as the value of $\theta$ increases, that is to say, increasing the chance of mating with sterilized mosquito reduces the spate in population of reproductive mosquitoes, consequently reducing the number of mosquito population in an environment. Fig. 8 depict total adult mosquitoes with different oviposition rate. 


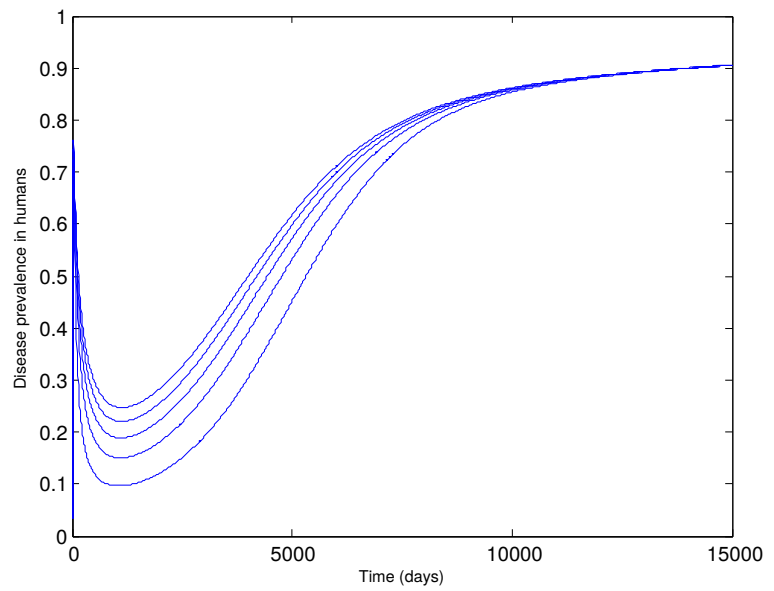

Fig. 5: Simulation of (5) showing disease prevalence in humans with different initial conditions and $R_{0}=24.7422$.

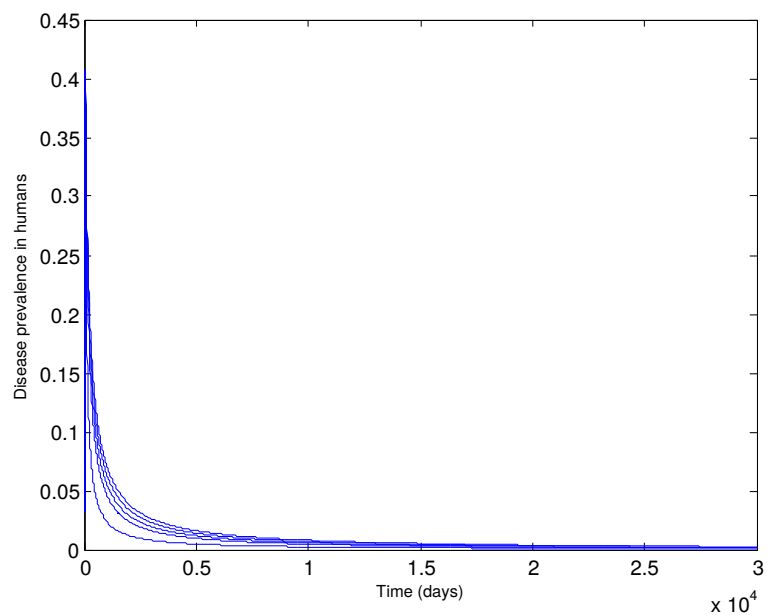

Fig. 6: Simulation of (5) showing disease prevalence in humans with different initial conditions and $R_{0}=0.5606$. 


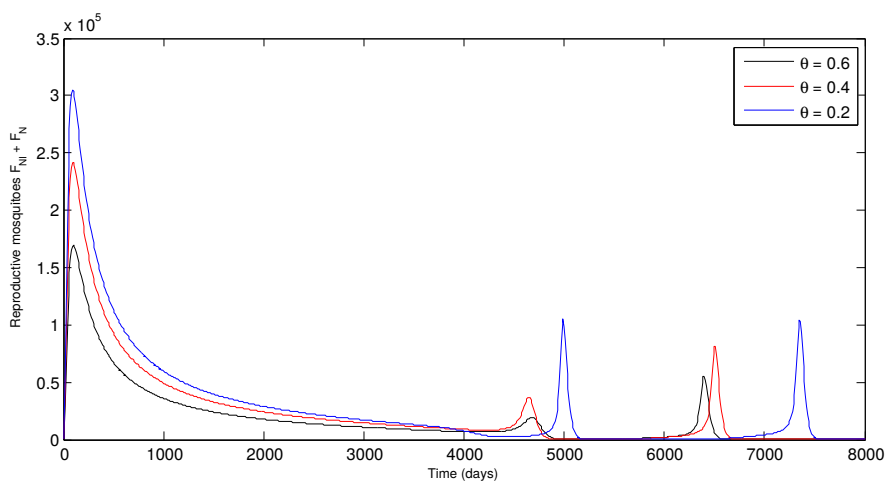

Fig. 7: Simulation of (5) showing the number of reproductive mosquitoes with $\theta=$ $0.2, \theta=0.4$ and $\theta=0.6$.

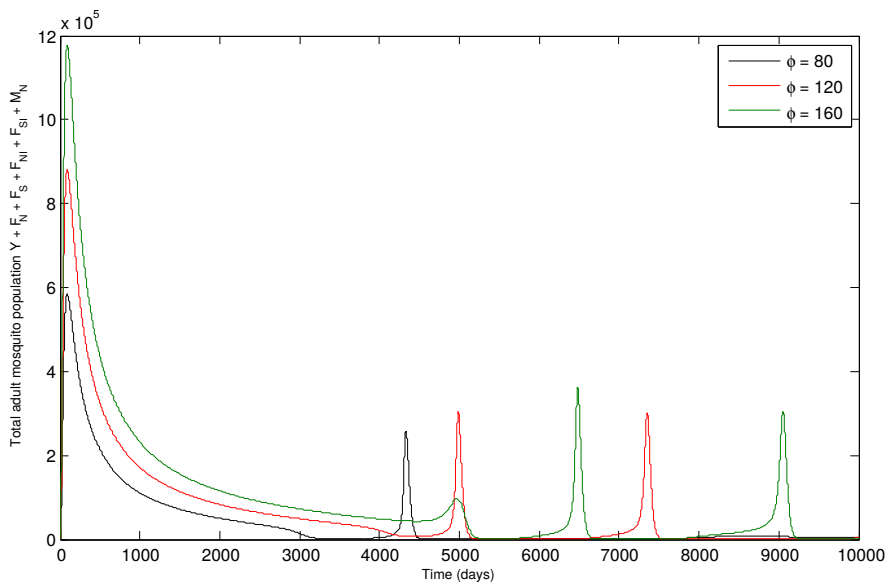

Fig. 8: Simulation of (5) showing the total number of adult mosquitoes with $\phi=80$, $\phi=120$ and $\phi=160$.

\section{Conclusion}

A Zika model where a fraction of male mosquitoes are being sterilized is studied. In addition to human-mosquito and mosquito-human transmission, the model which adopts a standard incidence formulation also allows direct (human-human) transmission. Both aquatic and non-aquatic stages of mosquito development were considered. The basic offspring number of the mosquito population was computed, as well as the basic reproduction number for the case when the basic offspring num- 
ber is greater than one. Using elasticity index (local sensitivity analysis), it is shown that, the most effective parameter for the control of the basic reproduction number in both areas of high and low transmission is mosquito death rate, while the adequate contact rate between infectious and susceptible humans is the most positively correlated parameter.

\section{Acknowledgment}

The authors acknowledge the support of DST-NRF Centre of Excellence in Mathematical and Statistical Sciences (CoE-MaSS) and the SARChI Chair on Mathematical Models and Methods in Biosciences and Bioengineering. Opinions expressed and conclusions arrived at are those of the author and are not necessarily to be attributed to the CoE-MaSS or the SARChI Chair. .

\section{References}

1. R. Anguelov, Y. Dumont and J. Lubuma. Mathematical Modeling of Sterile Insect Technology for Control of Anopheles Mosquito. Computers \& Mathematics with Applications, 64(3): 374389, 2012.

2. C. Bowman, A.B. Gumel, P. van den Drissche and J. Wu, H. Zhu. Mathematical model for assessing control strategies against West Nile virus. Bulletin of Mathematical Biology, 67: 1107-1133, 2005.

3. F. Brauer, C. Castillo-Chavez, A. Mubayi and S. Towers. Some models for epidemics of vector-transmitted disease. Infectious Disease Modelling; 1-9, 2016.

4. C. Castillo-Chavez, B. Song. Dynamical model of tuberculosis and their applications. Mathematical Bioscience and Engineering, 1(2): 361-404, 2004.

5. N. Chitnis, J.M. Hayman and J.M. Cushing. Determining important parameters in the spread of malaria through sensitivity analysis of a Mathematical model. Bulletin of Mathematical Biology, 70: 1272-1296, 2008.

6. N. Chitnis, J.M. Hayman and C.A. Manore. Modelling vertical transmission in vectore-borne diseases with application to Rift Valley fever. Journal of Biological Dynamics, 7(1): 11-40, 2013.

7. A. Clemons, A. Mori, M. Haugen, et al. Aedes aegypti Culturing and Egg Collection. Cold Spring Harb Protoc. 2010:pdb.prot5507.

8. F.A.B. Coutinho, M.N. Burattini, L.F. Lopez and E. Massad. Threshold conditions for a nonautonomous epidemic system describing the population dynamics of dengue, Bulletin of Mathematical Biology 68: 22632282, 2006.

9. D. Musso, C. Roche, E. Robin, T. Nhan, A. Teissier and V. Cao-Lormeau. Potential Sexual Transmission of Zika Virus. Emerging Infectious Diseases, 21: 2, 2015.

10. Diekmann O, Heesterbeek JA, Roberts MG. The construction of next-generation matrices for compartmental epidemic models. Journal of the Royal Society Interface. 2010;7:873-885.

11. C. Dufourd and Y. Dumont. Impact of environmental factors on mosquito dispersal in the prospect of sterile insect technique control. Computers and Mathematics with Applications, 66: 1695-1715, 2013.

12. Y. Dumont, F. Chiroleu and C. Domerg. On a temporal model for the Chikungunya disease: Modeling, theory and numerics. Mathematical Biosciences, 213: 80-91, 2008. 
13. Y. Dumont and F. Chiroleu. Vector control for the Chikungunya disease. Mathematical Bioscience and Engineering, 7(2): 313-345, 2010.

14. Y. Dumont and J.M. Tchuenche. Mathematical studies on the sterile insect technique for the Chikungunya disease and Aedes albopictus. Journal of Mathematical Biology, 65(25): 809854, 2012.

15. P. van den Driessche and J. Watmough. Reproduction numbers and sub-threshold endemic equilibria for compartmental models of the disease transmission. Mathematical Bioscience, 180: 29-48, 2002.

16. E. DOrtenzio, S. Matheron, Y. Yazdanpanah Y, et al. Evidence of Sexual Transmission of Zika Virus. New England Journal of Medicine, 374(22):2195-8, 2016. DOI: 10.1056/NEJMc1604449 PMID: 27074370

17. L. Esteva, H.M. Yang. Control of Dengue Vector by the Sterile Insect Technique Considering Logistic Recruitment. TEMA. Tendtncias em Matemtica Aplicada e Computacional, 7(2); 259268, 2006.

18. B.D. Foy, K.C. Kobylinski, J.L.C. Foy, B.J. Blitvich, A.T. da Rosa, A.D. Haddow, R.S. Lanciotti, and R.B. Tesh. Probable NonVector-borne Transmission of Zika Virus, Colorado, USA. Emerging Infectious Diseases, textbf17: 880882, 2011.

19. T. Frour, S. Miralli, B. Hubert, C. Splingart, P. Barrire, M. Maquart, I. Leparc-Goffart. Sexual transmission of Zika virus in an entirely asymptomatic couple returning from a Zika epidemic area, France, April 2016. Euro Surveill., 21(23): 30254, DOI: http://dx.doi.org/10.2807/15607917.ES.2016.21.23.30254, 2016.

20. D. Gao, Y. Lou, D. He, et al. Prevention and Control of Zika as a Mosquito-Borne and Sexually Transmitted Disease: A Mathematical Modeling Analysis. Scientific Reports, 6(28070), 2016.

21. S.M. Garba, A.B. Gumel and M.R. Abu Bakar. Backward bifurcation in dengue transmission dynamics. Mathematical Bioscience, 215: 11-25, 2008.

22. S.M. Garba and M.A. Safi. Mathematical Analysis of West Nile Virus Model with Discrete Delays. Acta Mathematica Scientia, 33B(5): 1439-1462, 2013.

23. E. B. Hayes. Zika Virus Outside Africa. Emerging Infectious Diseases, 59: 13471350. 2009.

24. A. Mathieu, H. Niel, M. Christiaan, B. Philippe. Dynamic Epidemiological Models for Dengue Transmission: A Systematic Review of Structural Approaches. PLoS One: 7(11), e49085, 2012.

25. O. Maxiana, A. Neufeld, E. J. Talis, L. M. Childs, and J. C. Blackwood. Zika virus dynamics: When does sexual transmission matter? Epidemics. 2017.

26. A.S.O. Melo, G. Malinger, R. Ximenes, P. O. Szejnfeld, S. Alves Sampaio. Zika virus intrauterine infection causes fetal brain abnormality and microcephaly: tip of the iceberg. Ultrasound in Obstetrics and Gynecology, 47: 6-7, 2016.

27. H. Nishiura, R. Kinoshita, K. Mizumoto, Y. Yasuda and K. Nah. Transmission potential of Zika virus infection in the South Pacific. International Journal of Infectious Diseases, 12019712, 2016.

28. G.A. Ngwa and W.S. Shu. A mathematical model for endemic malaria with variable human and mosquito populations. Mathematical and Computer modeling, 32: 747-763, 2000.

29. C.F. Olivia, M. Jacquet, J. Gilles, G. Lemperiere, P-O. Maquart, et al. The Sterile Insect Technique for Controlling Populations of Aedes albopictus (Diptera: Culicidae) on Reunion Island: Mating Vigour of Sterilized Males. PLoS ONE, 7: 11, e49414, 2012.

30. H.L. Smith, P. Waltman. The Theory of the Chemostat, Dynamics of Microbial Competition. Cambridge University press, 1995. 